\title{
A árvore da WONCA: tradução e adaptação cultural para português
}

Paulo Santos, ${ }^{1,2,3}$ Armando Brito de Sá, ${ }^{1,4}$ Luiz Santiago, ${ }^{1,5,6}$ Alberto Hespanhol ${ }^{1,2,3,7}$

\section{RESUMO}

Introdução: A árvore da WONCA é uma representação visual dos conceitos e definições básicas da especialidade de medicina geral e familiar, publicada pelo Collège de Médecine de Premier Recours suíço em 2004 e revista em 2011. Até ao presente momento não havia uma tradução portuguesa da versão de 2011 deste instrumento. O objetivo deste trabalho foi traduzir com uma adaptação cultural e validar a versão portuguesa da árvore da WONCA.

Métodos: Procedeu-se a uma análise de consenso partindo da versão em inglês da árvore da WONCA, aplicando uma metodologia sequencial em três passos: revisão e tradução inicial; processo de consenso por método Delphi; e avaliação e consenso final. Um grupo de 74 peritos da especialidade de medicina geral e familiar, entre os 128 convidados, participou na avaliação do texto traduzido em duas rondas até atingir a proporção de concordância pré-estabelecida de $80 \%$.

Resultados: Na primeira ronda a proporção de concordantes com as expressões propostas foi de $85,9 \%$ (64,8\% a 98,6\%). Oito das 24 expressões não atingiram o nível de concordância pré-estabelecido e foram reenviadas após correção, tendo atingido uma concordância de 83,1\% (80,0 a 87,7\%). A versão final obteve uma proporção global de concordância de 88,7\% (IC95\%: 87,1-90,2).

Conclusão: A linguagem científica é, por definição, técnica. A universalidade do inglês na publicação científica tem vantagens significativas ao permitir uma comunicação eficaz entre diferentes países, mas existem nuances culturais que, numa tradução literal, poderão não representar fielmente as características culturais e sociais de populações com outros idiomas. Esta tradução valida para a língua portuguesa a árvore da WONCA.

Palavras-chave: Medicina familiar; Medicina geral; Cuidados de saúde primários; Tradução.

\section{INTRODUÇÃO}

A medicina diferencia-se na capacidade de juntar sintomas e sinais num esquema de raciocínio clínico capaz de gerar uma ou mais hipóteses prováveis, de onde sai o diagnóstico definitivo, cuja abordagem específica da intervenção terapêutica vai alterar a história natural da doença e tendencialmente melhorar o respetivo prognóstico.

Esta é definição do ato médico. Baseia-se na perspetiva de que existe sempre um problema de saúde - a

\footnotetext{
1. Especialista em Medicina Geral e Familiar

2. Departamento de Medicina da Comunidade, Informação e Decisão em Saúde. Faculdade de Medicina, Universidade do Porto.

3. Centro de Investigação em Tecnologias e Servços de Saúde (CINTESIS). Universidade do Porto

4. USF Conde Saúde, ACeS da Arrábida.

5. USF Topázio, ACeS Baixo Mondego.

6. Clínica Universitária de MGF. Faculdade de Medicina, Universidade de Coimbra.

7. USF S. João, ACeS Porto Ocidental.
}

doença - que justifica a consulta médica, seja atual no diagnóstico, seja futuro na prevenção.

A doença assume um caráter tão relevante nesta relação do médico com o doente que leva à necessidade de diferenciação cada vez mais específica, no sentido de melhor tratar as anomalias que vão sendo detetadas. $\mathrm{O}$ aumento da capacidade tecnológica permitiu melhorar a acuidade diagnóstica e a especificidade terapêutica. Ao mesmo tempo aumentou a complexidade do raciocínio e tornou necessária a evolução para a especialização médica no sentido de ser possível acompanhar a explosão de conhecimento, sobretudo após a $2^{\mathrm{a}}$ Grande Guerra Mundial. Ohospital, centro de excelência para o tratamento das doenças, desenvolveu-se e aprimorou-se. O ambulatório, apesar de alguma indefinição nos processos organizativos, seguiu-lhe o rumo. Chegados aos anos 60, havia uma boa resposta face à doença, mas não uma resposta satisfatória 
face à saúde, numa inversão clara da pirâmide de necessidades onde a maior parte da população é saudável e apenas alguns necessitam de cuidados na doença. ${ }^{1}$

A subespecialização fragmenta a pessoa e coloca-a à mercê da avaliação parcelar do funcionamento dos seus órgãos, com critérios cada vez mais apertados para definição de anomalia. Neste contexto, a saúde é entendida como o contrário da doença e o saudável é aquele que não tem doença detetável num qualquer órgão. Cada departamento vela pelo seu órgão e garante a sua quota parte da saúde total.

O generalista parecia estar condenado a uma posição de segunda linha na abordagem do doente, como, aliás, Collings nota em 1950 na revisão que faz da clínica geral inglesa, ${ }^{2}$ num artigo publicado na Lancet e que viria a ser o mote fundador do Royal College of General Practitioners. Incorporam-se os trabalhos do todo biopsicossocial de Engel $^{3} \mathrm{e}$ da abrangência de Balint ${ }^{4} \mathrm{e}$ surge a oportunidade de recentrar a medicina na pessoa mais do que na doença, o que encaixava na necessidade que muitos sentiam de uma postura diferente no acompanhamento de proximidade e em continuidade, localmente e normalmente longe dos grandes hospitais tecnológicos, onde assumiam especial relevância características como a compreensão da individualidade do doente, a partilha do poder e da responsabilidade na saúde, a aliança terapêutica e o médico enquanto também ele pessoa. ${ }^{5}$

A definição de saúde remete para esta abrangência. Saúde é o completo bem-estar físico, mental, social e existencial e não apenas a ausência de doença. ${ }^{6}$ Mais recentemente, Huber e colaboradores foram mais longe quando abordaram a definição de saúde em termos da capacidade de autoadaptação às condições do meio, mantendo a homeostasia, introduzindo o conceito de ser saudável, apesar de estar doente. ${ }^{7}$

Privada de um órgão ou de uma doença que a defina enquanto corpo de conhecimento, a CLÍ́NICA GERAL, ou Medicina Geral, mais tarde também Familiar, começa por se definir na prestação de cuidados médicos pessoais, primários (essenciais), contínuos e acessíveis a indivíduos e famílias, ${ }^{8}$ com o objetivo de promover a saúde que considera no seu todo holístico. O grupo de Leuwenhorst, na Holanda (1974), consolida o conceito da pessoa para lá do incidente/doença e introduz o conceito dos cuidados em continuidade, embora episódicos na forma como se processa o acesso ao médico, in- dependentemente do sexo, idade ou problema de saúde, contribuindo para o estabelecimento de uma relação de confiança, que é por si só terapêutica se trabalhada de forma profissional. ${ }^{9}$ Em 2002, a WONCA Europa define as onze características da disciplina de clínica geral/medicina familiar ou, como se optou em Portugal, Medicina Geral e Familiar, agrupadas nas seis competências básicas de gestão de cuidados primários, orientados em função da comunidade envolvente, mantendo a centralidade dos cuidados na pessoa, com aptidão para a resolução de problemas específicos, a utilização de uma abordagem abrangente e a integração do modelo holístico. ${ }^{10}$ Esta versão foi revista no sentido de a tornar mais clara em 2005 e, novamente em 2011, com a introdução de uma $12^{\mathrm{a}}$ característica relacionada com a capacitação do doente para gerir a sua própria saúde. ${ }^{11}$

Em 2005, o Collège de Médecine de Premier Recours, da Suíça, publicou a árvore da WONCA, aproveitando a definição da especialidade de 2002 daWONCA Europa para um aspeto visual interessante capaz de resumir os princípios da especialidade. ${ }^{12}$ Esta versão foi traduzida para português e publicada na Revista Portuguesa de Clínica Geral..$^{13}$ Entretanto, com a revisão de 2011, foi revista também a árvore que, até ao momento, não foi traduzida.

O objetivo deste trabalho é apresentar uma tradução e adaptação cultural para português da versão de 2011 da árvore da WONCA (copyright 2011, Collège de Médecine de Premier Recours).

\section{MÉTODOS}

Realizou-se uma análise de consenso, partindo da versão em inglês da árvore da WONCA publicada pelo Colégio Suíço de Medicina dos Cuidados Primários. ${ }^{11} \mathrm{O}$ estudo foi conduzido em três passos sequenciais.

No primeiro passo, três autores reviram a árvore da WONCA e traduziram-na para português, enquadrando o texto original nos conceitos da Definição Europeia de Medicina Geral e Familiar.

No segundo passo, esta versão foi submetida a uma análise de consenso por um grupo de peritos. Foi pedido a todos que comentassem as diferentes frases ou simplesmente concordassem com a tradução proposta.

No terceiro passo, os comentários foram integrados nas frases que não atingiram a proporção de concordância desejada e nova proposta foi enviada ao grupo de peritos para análise. 


\section{Revisão e tradução inicial}

A aceitação da universalidade do inglês na comunicação científica tem vantagens significativas ao permitir uma comunicação eficaz entre diferentes países. Mas a linguagem científica é, por definição, técnica e não facilita a expressão idiomática e a aproximação cultural e social do texto à pessoa. Como em qualquer idioma, existem nuances culturais que, numa tradução literal, poderão não representar fielmente as características culturais e sociais de populações com outros idiomas. Quando se iniciou esta tradução decidiu-se estruturar os termos entre dois polos distintos, mas complementares: por um lado, manter a lógica do texto que se remete aos conceitos da Definição Europeia de Medicina Geral e Familiar (no original inglês, The European Definition of General Practice/Family Medicine); por outro, incluir o espírito e a liberdade semântica da língua portuguesa, procurando no contexto as palavras que melhor traduzam as ideias. O resultado foi um conjunto de 24 expressões que iriam ocupar os campos de texto da árvore da WONCA.

\section{Processo de consenso Delphi}

Um processo Delphi utiliza questionários estruturados e individuais para obter uma opinião de grupo de um painel de especialistas num determinado campo. ${ }^{14}$ Neste caso, utilizou-se um questionário de concordância para cada uma das 24 expressões resultantes do processo anterior. O painel de peritos incluiu um conjunto de 128 especialistas em medicina geral e familiar, com base na sua experiência enquanto médicos de família e na capacidade de conceptualização da especialidade nos seus princípios básicos e definições. Foi enviado o questionário a todos os associados da ADSO (Associação de Docentes e Orientadores de Formação em Medicina Geral e Familiar), aos professores universitários na área da medicina geral e familiar, aos membros do Colégio de Medicina Geral e Familiar da Ordem dos Médicos, aos editores da Revista Portuguesa de Medicina Geral e Familiar e aos responsáveis pelo internato médico da especialidade. A todos foi pedido que assinalassem a concordância com a expressão traduzida face ao original em inglês, bem como a proposta alternativa se não se sentissem satisfeitos com a tradução.

\section{Revisão e consenso}

Definiu-se uma proporção de concordância mínima de $80 \%$ para aceitação das expressões. Para o cálculo desta proporção considerou-se o total de participantes. As respostas não assinaladas foram consideradas como não concordantes para efeitos de análise. As expressões que obtiveram concordância superior foram fechadas na formulação inicial. Todos os comentários recebidos na ronda anterior foram considerados. Nas expressões com concordância inferior a $80 \%$ procurou-se integrar os comentários recebidos na reestruturação da expressão, mantendo a fidelidade aos conceitos da definição europeia e procurando aproximar a nova expressão às diferentes contribuições entretanto recebidas.

Um novo questionário de concordância foi enviado aos participantes que responderam na primeira ronda. Por se ter atingido a proporção de concordância previamente estipulada não se procedeu a mais nenhuma ronda, concluindo-se assim a validação da tradução.

Dado tratar-se de uma análise documental, sem intervenção direta ou indireta sobre pessoas, é dispensável o parecer prévio de uma Comissão de Ética para a Saúde. Não obstante, o compromisso de atuar eticamente é mais importante do que a Lei que o regula. ${ }^{15} \mathrm{Na}$ condução deste trabalho foram respeitados os princípios éticos para a investigação clínica, nomeadamente o respeito pela confidencialidade dos dados e dos voluntários que graciosamente integraram o painel de peritos que foi consultado. Foi também obtida autorização formal do Colégio Suíço de Medicina dos Cuidados Primários, titular da publicação original da árvore da WONCA. ${ }^{11-12}$

\section{RESULTADOS}

O projeto de tradução foi realizado entre março e maio de 2020. O número de participantes no painel Delphi foi de 74 na primeira ronda (taxa de resposta de $58 \%$ ) e de 65 na segunda ronda, correspondente a $88 \%$ do total de convites.

Na primeira ronda, a proporção de concordantes com as expressões propostas foi de 85,9\% (IC95\%: 84,2$87,5)$, variando entre $64,8 \%$ e $98,6 \%$. Oito das 24 expressões não atingiram o nível de concordância pré-estabelecido de $80 \%$ dos participantes (Tabela 1 ).

Na segunda ronda foi alterada a tradução das oito expressões e reenviada aos participantes que aceitaram responder à primeira ronda. A proporção de concordantes com as novas expressões foi de $83,1 \%$ (IC95\%: 79,6-86,2). Todas as expressões atingiram o nível de 


\begin{tabular}{|c|c|c|c|c|}
\hline Original & Tradução proposta ( $1^{\text {a }}$ fase) & Concordância & Tradução proposta ( $2^{\mathrm{a}}$ fase) & Concordância \\
\hline \multicolumn{5}{|c|}{ Base da árvore } \\
\hline Attitude & Atitude & $86 \%$ & & \\
\hline Science & Ciência & $93 \%$ & & \\
\hline Context & Contexto & $97 \%$ & & \\
\hline \multicolumn{5}{|c|}{ Tronco } \\
\hline Clinical tasks & Tarefas clínicas & $91 \%$ & & \\
\hline $\begin{array}{l}\text { Communication with } \\
\text { patients }\end{array}$ & Comunicação com os doentes & $79 \%$ & Comunicação & $82 \%$ \\
\hline $\begin{array}{l}\text { Management of the } \\
\text { practice }\end{array}$ & Gestão da prática clínica & $99 \%$ & & \\
\hline \multicolumn{5}{|c|}{ Competências básicas } \\
\hline Person-centred care & Cuidados centrados na pessoa & $78 \%$ & $\begin{array}{l}\text { Cuidados centrados na pessoa e } \\
\text { na família }\end{array}$ & $82 \%$ \\
\hline Community orientation & $\begin{array}{l}\text { Cuidados orientados para a } \\
\text { comunidade }\end{array}$ & $84 \%$ & & \\
\hline $\begin{array}{l}\text { Specific problem } \\
\text { solving skills }\end{array}$ & $\begin{array}{l}\text { Competências específicas de } \\
\text { resolução de problemas }\end{array}$ & $84 \%$ & & \\
\hline $\begin{array}{l}\text { Comprehensive } \\
\text { approach }\end{array}$ & Abordagem abrangente & $93 \%$ & & \\
\hline $\begin{array}{l}\text { Primary care } \\
\text { management }\end{array}$ & $\begin{array}{l}\text { Gestão de cuidados de saúde } \\
\text { primários }\end{array}$ & $93 \%$ & & \\
\hline Holistic modelling & Abordagem holística & $93 \% \%$ & & \\
\hline \multicolumn{5}{|c|}{ Competências específicas } \\
\hline $\begin{array}{l}\text { Doctor-patient } \\
\text { relationship }\end{array}$ & Relação médico-doente & $88 \%$ & & \\
\hline $\begin{array}{l}\text { Centred on patient and } \\
\text { context }\end{array}$ & $\begin{array}{l}\text { Centrados no doente e no } \\
\text { contexto }\end{array}$ & $78 \%$ & $\begin{array}{l}\text { Centrados na pessoa e nos } \\
\text { contextos }\end{array}$ & $80 \%$ \\
\hline $\begin{array}{l}\text { Promotes patient } \\
\text { empowerment }\end{array}$ & Promotores de capacitação & $69 \%$ & $\begin{array}{l}\text { Promotores da capacitação da } \\
\text { pessoa }\end{array}$ & $88 \%$ \\
\hline Longitudinal continuity & Cuidados longitudinais & $89 \%$ & & \\
\hline $\begin{array}{l}\text { Responsible for health } \\
\text { of the community }\end{array}$ & $\begin{array}{l}\text { Responsabilidade pela saúde da } \\
\text { comunidade }\end{array}$ & $91 \%$ & & \\
\hline $\begin{array}{l}\text { Decision making based } \\
\text { on incidence and } \\
\text { prevalence }\end{array}$ & $\begin{array}{l}\text { Decisão baseada em dados } \\
\text { epidemiológicos }\end{array}$ & $77 \%$ & $\begin{array}{l}\text { Decisão baseada na incidência e } \\
\text { prevalência }\end{array}$ & $86 \%$ \\
\hline $\begin{array}{l}\text { Early undifferentiated } \\
\text { stages }\end{array}$ & Estádios iniciais indiferenciados & $86 \%$ & & \\
\hline $\begin{array}{l}\text { Acute and chronic } \\
\text { health problems }\end{array}$ & $\begin{array}{l}\text { Problemas de saúde agudos e } \\
\text { crónicos }\end{array}$ & $99 \%$ & & \\
\hline $\begin{array}{l}\text { Promotes health and } \\
\text { wellbeing }\end{array}$ & Promoção da saúde e bem-estar & $92 \%$ & & \\
\hline $\begin{array}{l}\text { Care coordination and } \\
\text { advocacy }\end{array}$ & $\begin{array}{l}\text { Coordenação de cuidados e } \\
\text { advocacia }\end{array}$ & $76 \%$ & $\begin{array}{l}\text { Provedoria e coordenação de } \\
\text { cuidados }\end{array}$ & $80 \%$ \\
\hline $\begin{array}{l}\text { First contact, open } \\
\text { access, all health } \\
\text { problems }\end{array}$ & $\begin{array}{l}\text { Porta de entrada, acessibilidade, } \\
\text { todos os problemas de saúde }\end{array}$ & $65 \%$ & $\begin{array}{l}\text { Primeiro contato, acessibilidade, } \\
\text { todos os problemas de saúde }\end{array}$ & $82 \%$ \\
\hline $\begin{array}{l}\text { Physical, psychological, } \\
\text { social, cultural and } \\
\text { existencial }\end{array}$ & $\begin{array}{l}\text { Biopsicossocial, cultural e } \\
\text { espiritual }\end{array}$ & $80 \%$ & $\begin{array}{l}\text { Biopsicossocial, cultural e } \\
\text { existencial }\end{array}$ & $86 \%$ \\
\hline
\end{tabular}




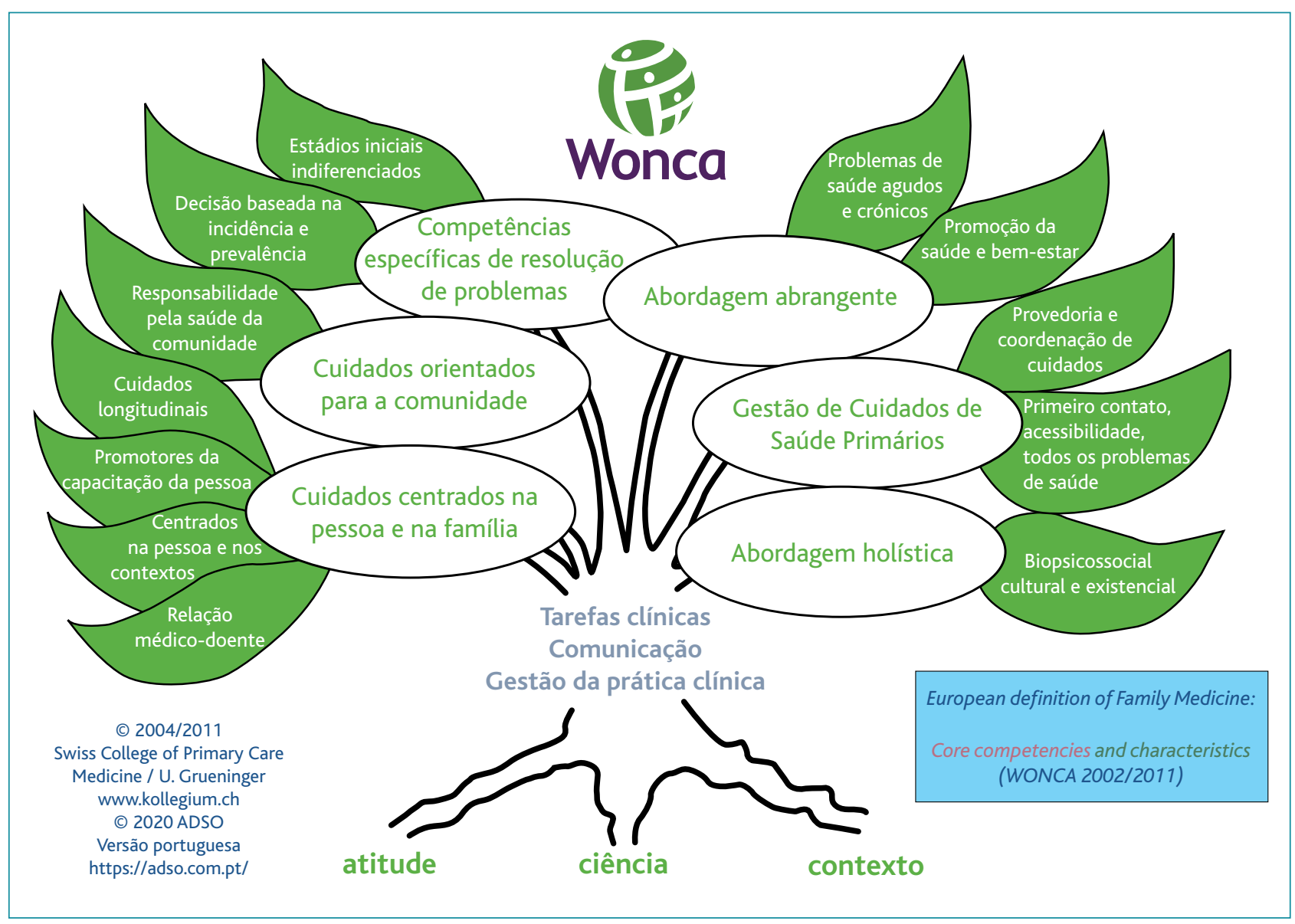

Figura 1. Versão portuguesa da árvore da WONCA, representando as competências e características da especialidade de Medicina Geral e Familiar (tradução do original do Collège de Médecine de Premier Recours, Suisse, 2004/2011).

concordância mínimo de $80 \%$, pelo que foi encerrado o processo de recolha de opinião.

$\mathrm{Na}$ análise da versão final obteve-se uma proporção global de concordância de 88,7\% (IC95\%: 87,1-90,2). A Figura 1 mostra a tradução portuguesa da árvore da WONCA, versão 2011.

\section{DISCUSSÃO}

A perceção da doença e o reconhecimento do seu potencial destruidor terão sido das primeiras manifestações de que o ser humano é um ser biológica e racionalmente mais diferenciado ${ }^{16} \mathrm{e}$ levaram à diferenciação da medicina enquanto corpo de conhecimento capaz de restabelecer a homeostasia face à entrópica doença. O ato médico surge, assim, em consequência do ser humano e para ele, aproveitando a constante evolução tecnológica para se aprimorar e melhorar. Na explosão de conhecimento da segunda metade do século XX deixou de ser possível a atualização constante na diversidade de doenças que poderiam estar presentes num determinado contexto, percebendo-se a necessidade de especialização em áreas de conhecimento. A pessoa foi, assim, espartilhada por órgãos, sistemas e fases da sua vida, entregue sectorialmente a cada especialista que trataria na vanguarda do conhecimento as doenças que apresentasse. Mas esta medicina da patologia deixava de fora uma parte significativa da população que não necessitava destes cuidados especializados, mas não podia prescindir da assistência médica. White, Williams e Greenberg mostram que esta diferenciação tecnocrática se destina a menos de $1 \%$ da população em risco, ${ }^{1}$ retomando a ideia de um médico generalista, agora mais atento à pessoa que o consulta, no 
seu contexto e nas suas necessidades de saúde e de doença, do que orientado para a doença enquanto eixo central dos cuidados que proporciona. ${ }^{4}$

Na evolução do pensamento, a definição do especialista em medicina geral e familiar, como designado em Portugal, assenta na formação médica, na abordagem generalista e na criação de uma disciplina ou corpo de conhecimento próprio, que carece de treino específico. ${ }^{11} \mathrm{O}$ médico de família exerce uma medicina personalizada, com respeito pela autonomia da pessoa que o procura, caracterizada por cuidados abrangentes e em continuidade, independentemente da idade, sexo, etnia ou problema de saúde, integrando o contexto familiar, comunitário e cultural, e reconhecendo a responsabilidade para com a comunidade. Integra o modelo holístico na partilha da decisão, utilizando a relação de conhecimento e confiança gerada por contactos repetidos. A sua prática engloba os aspetos preventivos, de tratamento, de reabilitação e de paliação, promovendo a capacitação e a autogestão da pessoa com quem partilha a responsabilidade pela sua saúde. Atua diretamente ou utilizando outros recursos da comunidade, da área da saúde ou fora dela, e auxilia a pessoa no acesso aos serviços necessários. Assume a necessidade de se manter atualizado e competente na sua atividade profissional e procura manter um equilíbrio pessoal para propiciar um atendimento eficaz e seguro. Na equipa que integra responsabilizase pelo controlo e melhoria contínua da qualidade, tanto nos aspetos clínicos do exercício como na organização, na segurança e na satisfação das pessoas que o procuram.

É uma definição extensa que procura traduzir a complexidade inerente ao exercício da medicina geral e familiar, centrada no indivíduo, mas integrada numa equipa alargada de profissionais dentro e fora da saúde. ${ }^{17} \mathrm{Na}$ comparação com outras especialidades, Katerndahl encontra maiores índices de complexidade na medicina geral e familiar, não pela diferenciação tecnológica, mas sobretudo pela diversidade inerente às pessoas e aos seus contextos e características, nomeadamente a gestão da pessoa com multimorbilidade. ${ }^{18-19}$

A árvore da WONCA é uma tentativa feliz de esquematizar esta definição, apresentando os conceitos numa estrutura visual de fácil leitura. A tradução agora proposta pretende ser fiel ao original da árvore e das definições que representa, enquanto insere uma aculturação portuguesa. Na maior parte das expressões a tradução é simples por estar já incorporada na raiz do exercício dos médicos de família.

Ainda assim, surgiram algumas questões que não ficam completamente resolvidas nesta tradução.

A primeira é como designar as pessoas que consultam os médicos de família. A expressão clássica «doente» deriva do passado em que a atuação médica se centrava na doença, enquanto patologia. Não se enquadra no comportamento atual dos médicos de família e, portanto, foi evitada. A exceção ficou na expressão «relação médicodoente», que parece estar consolidada e centrar-se mais na parte da relação do que na descrição dos participantes. Em sua substituição, doente é muitas vezes referido como utente que, na língua portuguesa, significa aquele que usa ou que tem o direito de usar. Esta expressão foi popularizada pelo Serviço Nacional de Saúde, enquanto instrumento de promoção do direito constitucionalmente garantido de acesso aos serviços de saúde, mas é redutora da interação que acontece na intimidade da consulta, onde ambos os intervenientes têm um papel ativo, com processos próprios e bem definidos, resultando numa interação em que o total é significativamente superior à soma das partes. Outro termo vulgarmente utilizado é paciente, uma palavra com origem latina patientem, que significa o que sofre, o que padece. É uma palavra aplicada àquele está sob o tratamento de um médico, mas ao mesmo tempo indica aquele que sabe esperar, sem pressa, o curso dos acontecimentos. Patient é a designação usual em língua inglesa, em que apresenta também um aspeto de personalização da abordagem: o meu paciente ou o paciente desta instituição. Apesar de ser um melhor tradutor da relação muito especial que estabelece com o médico remete para a doença/sofrimento e coloca as mesmas questões da palavra «doente». Optou-se, assim, por pessoa. Associada à personalidade física, mental, social e também jurídica, «pessoa» remete para a individualidade e responsabilidade e retira a carga associada à patologia, muitas vezes perfeitamente ausente nos encontros clínicos em medicina geral e familiar.

A segunda questão coloca-se em relação à tradução do termo original inglês advocacy, originado de uma linguagem jurídica que remete para o trabalho de defesa em tribunal. Na génese latina, advocatus é aquele que é chamado a prestar assistência a um acusado. Esta função de advogado deriva do compromisso ético e deontológico em que o médico assume que atua sempre na 
defesa dos melhores interesses das pessoas. No entanto, o espírito dos códigos é o do respeito pela dignidade do ser humano, prestando os melhores cuidados, agindo com correção e delicadeza, no intuito de promover ou restituir a saúde, conservar a vida e a sua qualidade, e suavizar os sofrimentos, ${ }^{20}$ incluindo uma ponderação entre os princípios éticos de Beauchamp ${ }^{21}$ e não uma defesa intransigente num jogo de meios de prova para obtenção da verdade decretada. Na realidade, o que está em causa nesta competência é providenciar um conjunto de conhecimentos e estratégias capazes de ajudar a pessoa no seu percurso pelos serviços de saúde, a fim de satisfazer as necessidades de saúde ou de doença, num contexto de provedoria e não de advocacia.

A terceira questão que fica em aberto é a tradução de empowerment. A palavra inglesa deriva da palavra «poder» e reflete o processo de cooperação com o médico que leva a pessoa a ganhar esse poder, atribuído ou compartilhado, para livremente fazer o que entenda, controlando o que acontece consigo próprio. ${ }^{22} \mathrm{~A}$ tradução para português em «empoderamento» remete para o mesmo significado relacionado com dar ou adquirir poder ou mais poder. No contexto da consulta de medicina geral e familiar, interessa a capacitação em que a pessoa adquire conhecimentos, atitudes e aptidões que lhe permitam lidar com os processos de saúde e de doença, melhorando a literacia em saúde, fator chave para uma decisão informada, livre e esclarecida. ${ }^{23}$

Neste tipo de estudos, a seleção do painel de peritos é muito importante para garantir uma representatividade em relação à população geral. Neste caso, selecionou-se um conjunto de especialistas em medicina geral e familiar com experiência clínica, e em muitos casos também académica, capazes de pensar as definições da especialidade e com uma vivência clínica que permite o ajustamento à realidade do exercício. A taxa de resposta foi satisfatória, o que é um ponto forte desta tradução, mas muitos outros médicos poderiam ter contribuído com sugestões valiosas para este processo de tradução. Não obstante, o resultado parece ser satisfatório com uma proporção de concordantes muito alta. Fica à disposição de todos a versão portuguesa da árvore da WONCA, conforme a versão de 2011 do Colégio Suíço de Medicina dos Cuidados Primários, que poderá constituir uma mais valia para o ensino e reflexão profissional da especialidade.

\section{AGRADECIMENTOS}

Um agradecimento à ADSO (Associação de Docentes e Orientadores de Formação de Medicina Geral e Familiar) que suportou cientificamente este trabalho.

Um agradecimento muito especial ao painel de peritos que aceitou colaborar e que concordou com a referência à sua participação neste artigo: Abílio Malheiro, Alexandre Rebelo Marques, Ana Rita Luz, Ana Sardinha, André Reis, André Tomé, Arancha Souto, Bruno Heleno, Bruno Melo, Carlos Franclim, Carlos Seiça Cardoso, Claudia Ho, Conceição Outeirinho, Cristina Ribeiro, Dagmara Paiva, Daniel Beirão, David Rodrigues, Débora Batista, Denise Velho, Diana Campos Lopes, Dina Gaspar, Dinis Brito, Foz Romão, Francisca Ribeiro Silva, Gabriela Amaral, Gaspar Caetano, Gisela Costa Neves, Gonçalo Envia, Helena Fragoeiro, Inês Rosendo, Isabel Nazaré, Isabel Santos, Isabela Carvalho, Ivo Reis, Jaime Correia de Sousa, Joana Silva Monteiro, João Sarmento, José Augusto Simões, José Eduardo Mendes, José Mendes Nunes, José Silva Henriques, Luciana Couto, Luís Alves, Luis Filipe Cavadas, Luis Rebelo, Luísa Sá, Maria do Rosário Gonçalves, Mariana Rio, Marta Dora Ornelas, Marta Magalhães, Nelson Rodrigues, Nuno Rodrigues, Pascale Charondière, Paulo Simões, Pedro Augusto Simões, Pedro Santos Sousa, Pedro Silva Almeida, Pimenta Marinho, Raquel Braga, Raquel Castro, Raquel Meireles, Rui Cernadas, Rui Lourenço, Rui Neto Fernandes, Rute Teixeira, Susana Pires da Silva, Teresa Rebello Andrade, Tiago Flores, Tiago Maricoto, Tiago Taveira-Gomes, Victor Ramos.

\section{REFERÊNCIAS BIBLIOGRÁFICAS}

1. White KL, Williams TF, Greenberg BG. The ecology of medical care. N Engl J Med. 1961;265:885-92.

2. Collings JS. General practice in England today: a reconnaissance. Lancet. 1950;255(6604):555-85.

3. Engel GL. The clinical application of the biopsychosocial model. J Med Philos. 1981;6(2):101-23.

4. Balint M. The doctor, his patient and the illness. 2nd ed. London: Pitman Paperbacks; 1974.

5. Mead N, Bower P. Patient-centredness: a conceptual framework and review of the empirical literature. Soc Sci Med. 2000;51(7):1087-110.

6. Constitution of the World Health Organization. Public Health Rep. 1946;61:1268-79.

7. Huber M, Knottnerus JA, Green L, van der Horst $H$, Jadad AR, Kromhout D, et al. How should we define health? BMJ. 2011;343:d4163.

8. Starfield B. Is primary care essential? Lancet. 1994;344(8930):112933.

9. Bentzen N, Boelaert RB, Borchgrevink CF, Byrne PS, Haeussler S, Heller $\mathrm{G}$, et al. The general practitioner in Europe: a statement by the working party appointed by the second European Conference on the Teaching of General Practice [Internet]. Leuwenhorst; 1974. Available from: https://euract.woncaeurope.org/sites/euractdev/files/documents/archive/publications/general-practitioner-europe-statement-workingparty-appointed-2nd-european-conference-teaching.pdf

10. Allen J, Gay B, Crebolder H, Heyrman J, Svab I, Ram P. The European definitions of the key features of the discipline of general practice: the role of the GP and core competencies. Br J Gen Pract. 2002;52(479): 526-7.

11. Allen J, Gay B, Crebolder H, Heyrman J, Svab I, Ram P. The European definition of GP/FM [homepage]. Wonca; 2011. Available from: 
https://www.woncaeurope.org/page/definition-of-general-practice-family-medicine

12. Grüninger U. Braucht es den Hausarzt noch? [Do we still need family physicians?] Prim Hosp Care. 2005;5(3):58-62. German

13. Allen J, Gay B, Crebolder H, Heyrman J, Svab I, Ram P, et al. A definição europeia de medicina geral e familiar (clínica geral/medicina familiar) [The European definition of family medicine (general practice/family medicine)]. Rev Port Med Geral Fam. 2005;21(5):511-6. Portuguese

14. Adler M, Ziglio E. Gazing into the oracle: the Delphi method and its application to social policy and public health. London: Jessica Kingsley Publishers; 1996. ISBN 9781853021046

15. Santos P, Teixeira P, Beça H, Hespanhol A. Evaluation of the research protocol by ethical committee [Internet]. In: Ray S, editor. Evidencebased medicine. London: IntechOpen; 2020. Available from: https:// www.intechopen.com/online-first/evaluation-of-the-research-protocol-by-ethical-committee

16. Namora F. Deuses e demónios da medicina. $2^{\text {a }}$ ed. Lisboa: Arcádia; 1958.

17. Lam CL. The role of the family doctor in the era of multi-disciplinary primary care. Fam Pract. 2016;33(5):447-8.

18. Prazeres F, Santiago L. The knowledge, awareness, and practices of Portuguese general practitioners regarding multimorbidity and its management: qualitative perspectives from open-ended questions. Int J Environ Res Public Health. 2016;13(11):1097.

19. Prazeres F, Santiago LM, Pereira PM, Santos PM, Cortinhal T. Multimorbilidade em medicina geral e familiar: construção e validação do Questionário de Avaliação da Sobrecarga da Gestão da Multimorbilidade em
Medicina Geral e Familiar (SoGeMM-MGF) [Multimorbidity in general and family medicine: construction and validation of the Questionnaire of Evaluation of Burden of Management of Multimorbidity in General and Family Medicine]. Gaz Med. 2019;6(4). Portuguese

20. Regulamento n. ${ }^{\circ} 707 / 2016$, de 21 de julho. Diário da República. $2^{\text {a Sé- }}$ rie(139).

21. BeauchampTL, Childress JF. Principles of biomedical ethics. 8th ed. New York: Oxford University Press; 2019. ISBN 9780195335705

22. World Health Organization. WHO guidelines on hand hygiene in health care: first global patient safety challenge clean care is safer care. Geneva:WHO; 2009.

23. Santos P, Sá L, Couto L, Hespanhol A. Health literacy as a key for effective preventive medicine. Cogent Soc Sci. 2017;3(1):1407522.

\section{CONFLITO DE INTERESSES}

Paulo Santos é editor-adjunto da RPMGF;Alberto Hespanhol é editor-chefe da RPMGF; os autores não apresentam qualquer conflito de interesses suscetível de interferir nos resultados deste estudo.

\section{ENDEREÇO PARA CORRESPONDÊNCIA \\ Paulo Santos \\ E-mail: psantosdr@med.up.pt \\ https://orcid.org/0000-0002-2362-5527}

Recebido em 25-07-2020

Aceite para publicação em 07-10-2020

\section{ABSTRACT}

\section{THE WONCA TREE: PORTUGUESE TRANSLATION AND CULTURAL ADAPTATION}

Introduction: The WONCA tree is a visual representation of the basic concepts and definitions of the specialty of general and family medicine, published by the Swiss Collège de Médecine de Premier Recours in 2004 and revised in 2011. No Portuguese translation of its 2011 version has been available until now. The aim of this study was to translate, providing a cultural adaptation, and to validate the Portuguese version of the WONCA tree.

Methods: We performed a consensus analysis based on the English version of the WONCA tree, applying a sequential three steps methodology: review and initial translation; consensus process using the Delphi method; and evaluation and final consensus. A group of 74 experts, all specialists in general and family medicine, among 128 invited, participated in the evaluation of the translated text in two rounds until reaching a pre-established agreement proportion of $80 \%$.

Results: In the first round the agreement proportion with the proposed expressions was $85.9 \%$, (64.8\% to $98.6 \%$ ). Eight of the 24 expressions did not reach the pre-established level of agreement and were resent after correction, reaching an agreement of $83.1 \%$ ( 80.0 to $87.7 \%)$. The final version obtained an overall proportion of agreement of $88.7 \%$ (95\%Cl: $87.1-90.2)$.

Conclusion: Scientific language is foremostly technical. Although the universality of English in scientific publishing has significant advantages, allowing effective communication between different countries, there are cultural peculiarities that may not accurately represent the cultural and social characteristics of populations with other languages in a literal translation. This translation validates the WONCA tree for the Portuguese language.

Keywords: Family practice; General practice; Primary health care; Translation. 